\title{
Automatic Quantification of Left Ventricular Systolic Wall Thickening Using Two-Dimensional Strain Assessed by a Novel Tissue-Tracking System
}

\author{
Renan Sukmawan, MD, Nozomi Watanabe, MD, Takashi Akasaka, MD, \\ Yoji Neishi, MD, Reishi Izumi, RDCS, Takahiro Kawamoto, MD \\ and Kiyoshi Yoshida, MD \\ Department of Cardiology, Kawasaki Medical School, Kurashiki, Japan
}

\begin{abstract}
Background. A novel tissue-tracking system (TTS) that enables automatic assessment of two-dimensional (2-D) strain of left ventricle (LV) segments simultaneously from gray-scale digital echocardiography images has been validated in an animal study at our institution. We sought to define the application of TTS for automatic quantification of systolic wall thickening (WT) with a study in humans.

Methods. We assessed 202 LV segments from 101 good and fair quality digital echo images acquired from 35 subjects. Radial strain was automatically derived from displacement between two points of interest placed at the epi- and endocardial borders of each segment. Peak radial strain at each segment was compared with manually measured systolic wall thickening.

Results. There were good correlation and agreement between 2-D radial strain assessed by TTS and manually measured systolic WT in overall subjects $(r=0.89, p<0.001)$. The correlation and agreements were better in good quality images $(r=0.94, p<0.001)$ rather than that in fair quality images $(r=0.82, p<0.001)$.

Conclusion. The novel TTS provides a method for automatic quantification of human LV systolic wall thickening.
\end{abstract}

(J Echocardiogr 2005; 3: 27-32)

Key words: tissue-tracking system, 2-D strain

\section{Introduction}

Routine methods of regional myocardial functional assessment in two-dimensional (2-D) echocardiography are mostly performed by wall motion observation based on the visual interpretation of endocardial excursion, which is subjective and at best semiquantitative [1-3]. Percent of systolic wall thickening (\%WT) had been introduced and widely used as a quantitative index to assess the left ventricle (LV) regional wall function [4-6]. Currently myocardial strain has been introduced as an emerging quantitative index in echocardiography. Basically, wall thickening is a radi-

Received January 28, 2005; revision received February 25, 2005; accepted March 14, 2005

Address for correspondence: Renan Sukmawan, MD

Department of Cardiology, Kawasaki Medical School,

577 Matsushima, Kurashiki 701-0192, Japan.

Telephone: +81-86-462-1111

Fax: +81-86-464-4060

E-mail: renan@med.kawasaki-m.ac.jp

(C) 2003 Journal of Echocardiography al strain measure that is used often as a parameter of regional contractile function [7]. However, the currently available myocardial strain assessment is derived from tissue Doppler imaging, which has limitations on its Doppler angle-beam dependency and is sometimes noisy [8].

A novel computerized tissue-tracking system (TTS) that enables automatic tracking of displacement between two points of interest within myocardial tissues from gray-scale 2-D digital echo image files has been developed and validated in an open-chest dog study at our institution [9]. This non-tissue Doppler system automatically generates strain profiles from the change in distance between the two points over time in an entire cardiac cycle. The clinical application of TTS in human subjects remains to be defined. In the present study we sought to define the application of the novel system for systolic WT quantification by comparing 2-D radial strain assessed by TTS and manually measured \%WT at the same LV segments in human subjects. 


\section{Methods}

We assessed digital echo images scanned from 35 consecutive subjects, comprising of patients with wall motion abnormalities in one or more segments and healthy subjects. Among the subjects (ages range $19-$ 78 years old, 28 male), 25 patients had a history of coronary artery diseases, and 10 were normal. We excluded patients with unstable conditions. All participants gave informed consent to the protocol approved by the committee for the protection of subjects in research at Kawasaki Medical School.

\section{Images acquisition.}

Two-D echo examination was performed using the Hitachi EUB-8500 (Hitachi Medical Corp, Japan) with a 2 - $4 \mathrm{MHz}$ wide band sector transducer. The images were acquired from 3 standard views: apical long-axis, apical four-chamber, and short-axis. After optimizing the gain, dynamic range, and time gain compensation, images were digitally recorded at frame rate ranges from 66 to 79 frame/s and were stored in magneto-optical discs. Two experienced observers classified the images into 3 categories based on image quality: good, fair, and poor. Images that clearly visualized both endo- and epicardial borders were categorized into good; less clear visualization but still recognizable were fair; and poor if one or more borders could not be recognized at all. The poor images were excluded from further data analysis.

\section{Two-dimensional radial strain assessment using} TTS.

In this study, the points of interest were placed at endo- and epicardial borders of LV segments. Two independent observers performed analysis by using the TTS workstation's personal computer equipped with customized analysis software. The TTS software is a 'pattern matching' algorithm that had been developed using a Matlab program (Mathworks company, Natick, Massachusetts). The algorithm searches the points in the following frames, which were assumed to be the 'matching' part for the selected points in the first frame, according to the information of pixel intensity distribution. The system tracks the distance change between the pair of points placed at LV segments and automatically generates a 2-D radial strain graph between the two points in all selected LV segments simultaneously (Figure 1). The distance between the selected points is defined as:

$$
\mathrm{d}_{i, j}(t)=\left|\mathrm{p}_{i}(t)-\mathrm{p}_{j}(t)\right|
$$

Where $d_{i, j}$ is the distance at the time $t$ and $\mathrm{p}_{i} \& \mathrm{p}_{j}$ are the coordinates of the first and second selected points
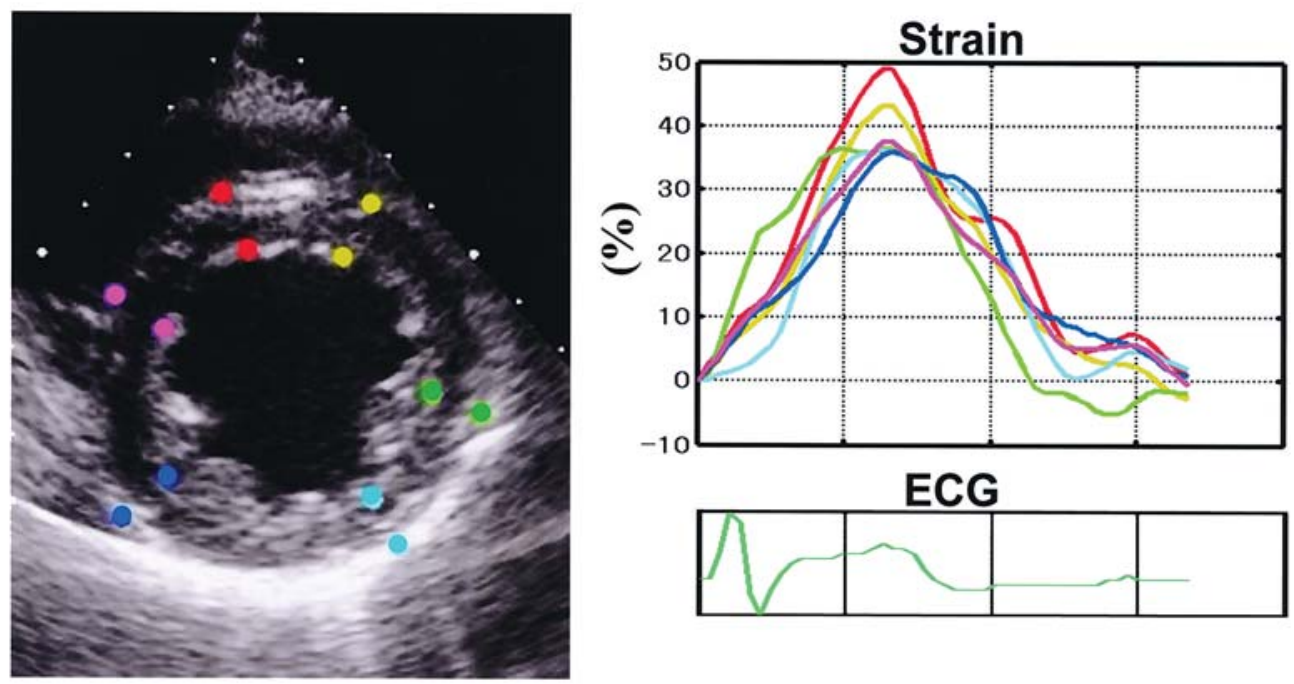

\section{Tissue-Tracking System}

Fig. 1. Illustration of 2-dimensional strain assessment by automated tissue-tracking system. Two points were placed at the epi- and endocardial borders of each LV segment (left) that result in the strain profiles in an entire cardiac cycle (right). 
respectively.

The algorithm determined strain at the time $t$ as follows:

$$
\operatorname{Strain}_{i, j}(t)=\frac{\mathrm{d}_{i, j}(t)-\mathrm{d}_{i, j}\left(t_{o}\right)}{d_{i, j}\left(t_{o}\right)}
$$

With $t_{o}$ as the initial time at the beginning of the cardiac cycle.

Distance resolution of the system is $0.1 \mathrm{~mm}$. To obtain strain value at each frame, the graph was converted to Excel data using Microsoft Excel software (Microsoft Corp, Redmond, Washington). The value of strain could also be obtained by directly clicking the computer mouse at selected sites within the graph.

\section{Calculation of \% WT manually.}

Two experienced observers, who did not know clinical data of the subjects, performed \%WT calculation manually. Maximum wall thickness at systole and minimum thickness at diastole were measured using measurement tools of the echo machine. The \%WT was calculated as: (maximum thickness - minimum thickness) X 100\% / minimum thickness [4, 10, 11].

\section{Correlation and agreement analysis.}

We assessed the relation between radial strain assessed by TTS and manually measured \%WT from 2 segments in each digital image: mid-anteroseptum and mid-inferolateral from the long-axis view; mid-inferoseptum and mid-anterolateral from the four-chamber view; and mid-anterior and mid-inferior from the shortaxis view. Correlation and agreement plots between 2$\mathrm{D}$ radial strain and manually measured \%WT in overall segments were made. The same plots were also made for segments with good and fair quality images respectively.

\section{Reproducibility of radial strain assessed by TTS.}

Two independent observers assessed radial strain using TTS at the same segment (mid-inferoseptum in four-chamber view) with good quality echo images from 10 randomly selected subjects in order to define interobserver variability. Similarly, intraobserver variability was obtained from two radial strain assessments in each 10 randomly selected subjects conducted by single observer.

\section{Statistical Analysis.}

All data are expressed as mean \pm SD for descriptive statistics. Correlation between radial strain and manually measured \%WT were plotted by regression analysis with the least-square method. The degree of agreement between radial strain and manually measured
\%WT was analyzed using the Bland and Altman method [12]. Inter- and intraobserver variability of radial strain assessment by TTS were plotted and analyzed by similar methods. A p-value $<0.05$ was considered significant.

\section{Results}

There were 105 echo images acquired from all subjects in this study with 55 images categorized as good quality, 46 in fair, and 4 in poor. The poor images were excluded from further analysis. Thus, TTS was applicable for most of the study patients in which 202 segments from 101 out of 105 images (96\%) were analyzed. Among them, 110 segments were obtained from 55 good echo quality images and 92 segments from 46 fair quality images.

\section{Systolic WT assessment.}

Regression analysis showed good correlation and agreement between 2-D radial strain assessed using TTS and manually measured \%WT in overall segments $(r=0.89, p<0.001$; mean difference $-0.50 \pm 13.09 \%$; Figure 2A). Better correlation and agreement were shown in good quality echo images $(r=0.94, p<0.001$; mean difference $0.86 \pm 10.2 \%$; Figure $2 \mathrm{~B}$ ). Meanwhile, correlation in the fair quality image was fair with wider difference in agreement analysis $(r=0.82, \mathrm{p}<0.001$; mean difference $-0.06 \pm 16.78 \%$; Figure $2 \mathrm{C}$ ).

\section{Reproducibility.}

There were good correlation and agreement of radial strain assessed using TTS between 2 independent observers $(\mathrm{r}=0.97, \mathrm{p}<0.001$; mean difference $-0.43 \pm 6.3 \%$; Figure $3 \mathrm{~A}$ ). Similarly, good correlation and agreement were obtained between two radial strain assessments at the same segment by a single observer $(\mathrm{r}=0.99, \mathrm{p}<0.001$; mean difference $-0.26 \pm 3.7$ $\%$; Figure $3 \mathrm{~B})$.

\section{Discussion}

The present study introduced the application of a newly developed TTS, which enables automatic assessment of 2-D regional myocardial strain at LV segments simultaneously, for quantification of systolic wall thickening. Actually, by placing the 2 arbitrary points of interest at appropriate directions in the LV wall, one may assess longitudinal, circumferential, or radial 2-D strains. The strains can be measured anywhere within the myocardium both in the endocardial or epicardial regions. For this study we placed the 2 points in the radial direction, at epi- and endocardial borders respec- 
A. Overal

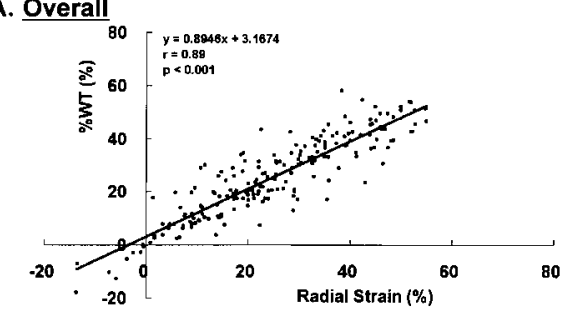

B. Good

$80 \quad y=0.9437 x+24400$
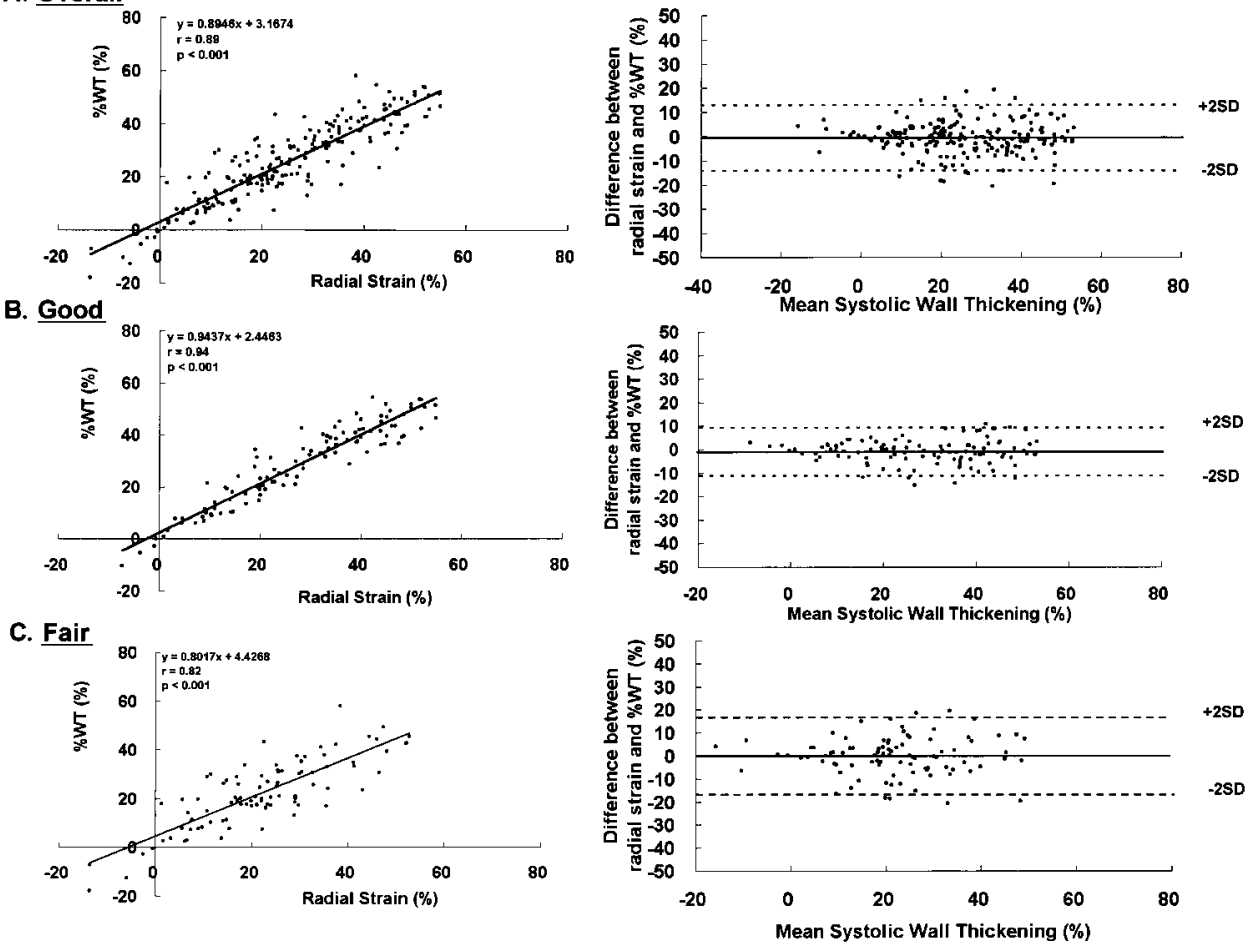

Fig. 2. Relation between radial strain and manually measured systolic wall thickening. Good correlation and agreement in overall segments $(A)$, with better correlation and agreement in good quality images (B), whereas fair correlation and wider difference on agreement analysis in fair quality images (C).

A. $z^{60}[y=1.0246 x-0.2861$

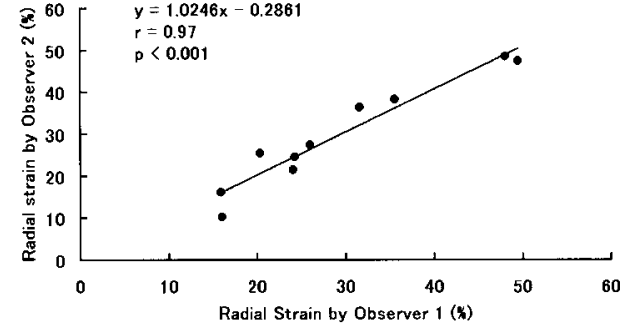

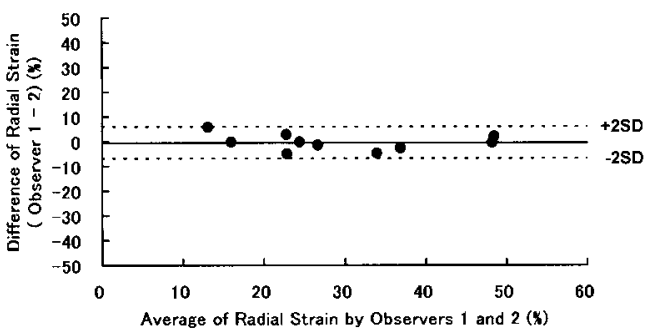

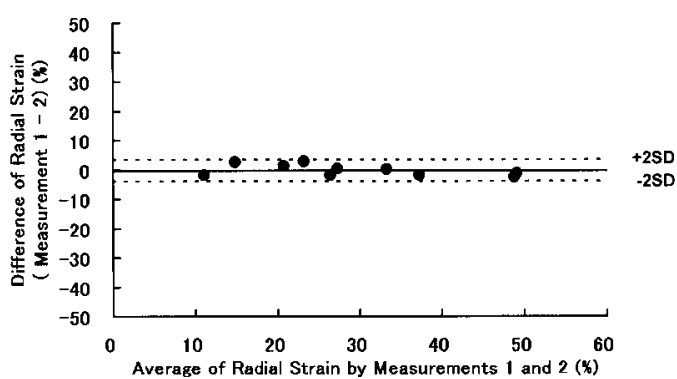

Fig. 3. Reproducibility of 2-dimensional radial strain assessment by TTS. Good correlation and agreement are shown in both interobserver $(A)$ and intraobserver $(B)$ variability analysis. 
tively, so that it was equivalent with an assessment of systolic wall thickening. We showed good correlation and agreement between radial strain assessed using TTS and manually measured \%WT especially in good quality echo images.

\section{Quantification of regional wall motion.}

Quantification of regional wall function had been the subject of many studies within the last two decades. Manually calculated systolic WT reported for the first time by Feneley et al [4]. The \%WT had been reported as a reliable and useful index in various clinical practices that may be obtained by either 2-D or M-mode echo $[13,14]$. Some other non-echocardiographic studies were also conducted to measure the systolic WT $[5,6,15]$. However, most of the studies were done manually and were time consuming. Those limitations have raised the need of an automatic computerized system for systolic WT quantification.

\section{Comparison with other regional wall motion studies.}

Computer-aided approach for quantification of regional LV function by assessing color mode echo has been reported [16] as well as other quantification methods by color kinesis. Those methods used color mode echocardiography that could not clearly provide information on epicardial motions $[17,18]$. They were different to our newly developed method that can be used to assess strain in gray-scale 2-D echo images and provide comprehensive information of endo- and epicardial motions. Another computer-assisted method has also been reported by using gated methoxyisobutylisonitrile single photon emission computed tomography (SPECT) [19]. However, SPECT has limitations in terms of space requirements and portability compared with echocardiography. Moreover a radiation issue may limit the application of SPECT. A computerized \%WT measurement in 2-D echo based on an integrated back-scatter system had also been introduced [11]. However, that system could only analyze a single segment at a time. The TTS in this study enables multisegment assessment simultaneously.

\section{Clinical Implication.}

The present study revealed the usefulness of TTS to assess \%WT automatically. Information of \%WT from all segments in a view could be obtained simultaneously. Since TTS has a distance resolution as high as 0.1 $\mathrm{mm}$, that is obviously a better resolution than ball-eye, we presumed that this might have potential clinical applications such as in dobutamine or exercise stress- echo. Further studies are required to define the benefits of this newly developed TTS in daily clinical practice.

\section{Limitations.}

As shown here, the TTS has better correlation and agreement with the manual method for systolic WT quantification, especially in good quality echo images. Once an error on automatic assessment occurs at the first frame, it may propagate to the following frames that result in incorrect strain values [9]. Thus, current application of TTS requires good quality 2-D echo images for optimal results of LV regional wall motion assessment. Moreover, to get optimal results of systolic WT measurement, it is necessary to put the 2points direction perpendicular to the cardiac wall. Currently a guiding marker feature has been added in the machine to assist the user in placing the 2-points perpendicular to the cardiac wall.

\section{Conclusion}

The novel tissue-tracking system provides a method for automatic quantification of human left ventricle systolic wall thickening.

\section{Acknowledgment}

We thank the technical assistance given by Hirotaka Baba, M.Eng. of Hitachi Medical Corp., Japan.

\section{References}

1. Visser CA, Kan G, Lie K, Becker AE, Durer D. Apex two dimensional echocardiography: alternative approach to quantification of acute myocardial infarction. Br Heart J 1982; 47: 461-7.

2. Parisi AF, Moynihan PF, Folland ED, Feldman CL. Quantitative detection of regional left ventricular contraction abnormalities by two-dimensional echocardiography, II: accuracy in coronary artery disease. Circulation 1981; 63(4) 761-7.

3. Moynihan PF, Parisi AF, Feldman CL. Quantitative detection of regional left ventricular contraction abnormalities by two-dimensional echocardiography, I: analysis of methods. Circulation 1981; 63(4): 752-60.

4. Feneley MP, Hickie JB. Validity of echocardiographic determination of left ventricular systolic wall thickening. Circulation 1984; 70: 226-32.

5. Jakob M, Hess OM, Jenni R, Heywood JT, Grimm J. Determination of left ventricular systolic wall thickness by digital subtraction angiography. Eur Heart J 1991; 53: 45-54.

6. Pflugfelder PW, Sechtem UP, White RD, Higgins CB. 
Quantification of regional systolic function by rapid cine MR imaging. Am J Roentgenol 1988; 150: 523-9.

7. Bogaert J, Rademakers E. Regional nonuniformity of normal adult human left ventricle. Am J Physiol Heart Circ Physiol 2000; 280: 610-20.

8. Urheim S, Edvardsen T, Torp T, Angelsen B, Smiseth OA. Myocardial strain by Doppler echocardiography: validation of a new method to quantify regional myocardial function. Circulation 2000; 102: 1158-64.

9. Toyoda T, Baba H, Akasaka T, Akiyama M, Neishi Y, Tomita J, et al. Assessment of regional myocardial strain by a novel automated tracking system from digital image files. J Am Soc Echocardiogr 2004; 17: 1234-8.

10. Myers JH, Striling MC, Choy M, Buda AJ, Gallagher KP. Direct measurement of inner and outer wall thickening dynamics with epicardial echocardiography. Circulation 1986; 74(1): 164-72.

11. Mizushige K, Furumoto W, Hirao K, Iwado Y, Ohmori K, Matsuo H. Quantitative evaluation of left ventricular regional wall motion using a real-time wall thickness curve system with two-dimensional echocardiography. Am J Cardiol 1999; 84: 1204-8.

12. Bland MJ, Altman DG. Statistical methods for assessing agreement between two methods of clinical measurement. Lancet 1986; 1: 307-10.

13. Borges AC, Pingitore C, Cordovil A, Sicari R, Baumann $\mathrm{G}$, Picano E. Heterogeneity of left ventricular regional wall thickening following dobutamine infusion in normal subjects. Eur Heart J 1995; 16(11): 1726-30.

14. Chan J, Wahi S, Cain P, Marwick TH. Anatomical Mmode: A novel technique for the quantitative evaluation of regional wall motion analysis during dobutamine echocardiography. Int J Card Imaging 2000; 16(4): 24755.

15. Sehgal M, Hirose K, Reed JE, Rumberger JA. Regional left ventricular wall thickness and systolic function during the first year after index anterior wall myocardial infarction: serial effects of ventricular remodeling. Int J Cardiol 1996; 53(1): 45-54.

16. Sklenar J, Jayaweera AR, Kaul S. A computer-aided approach for quantification of regional left ventricular function using two-dimensional echocardiography. J Am Soc Echocardiogr 1992; 5(1): 33-40.

17. Lang RM, Vignon P, Weinert L, Bednarz J, Korcarz C, Sandelski RDMS J, et al. Echocardiographic quantification of regional left ventricular wall motion with color kinesis. Circulation 1996; 93(1): 1877-85.

18. Mor-Avi V, Vignon P, Koch R, Weinert L, Garcia MJ, Spencer. Segmental analysis of color kinesis images: New method for quantification of the magnitude and timing of endocardial motion during left ventricular systole and diastole. Circulation 1997; 95; 2082-97.

19. Zhang JJ, Raichlen JS, Kim SM, Manief AR, Intenzo CM. Computer-assisted myocardial thickening analysis of gated MIBI SPECT images. Invest Radiol 1998; 33(5): 257-62. 